\section{Estado nutricional e sua relação com fatores biológicos, sociais e demográficos de crianças assistidas em creches da Prefeitura do Município de São Paulo}

\section{Nutritional status and relationship with biological, social and demographical issues of children attending daycare centers of the local government of the city of São Paulo}

Cristina Carpentieri Zöllner 1 Regina Mara Fisberg 2

1 Coordenadoria da Área Técnica de Alimentação e Nutrição. Secretaria de Estado da Saúde do Espírito Santo. Vitória, ES, Brasil.

2 Departamento de Nutrição. Faculdade de Saúde Pública. Universidade de São Paulo. Av. Dr. Arnaldo, 715. Cerqueira César. São Paulo, SP, Brasil. CEP: 01.246-904.

\begin{abstract}
Objectives: to describe the factors associated to the children's nutritional state assisted in day-care centers of the City hall of São Paulo Municipal district.

Methods: a probabilistic sample of 556 children between 4 and 84 months old was selected. The National Center of Health Statistics growth curve was used as the reference for the weight/age, weight/height and height/age indices. Children with indices two $z$ scores below the median value of the reference population were considered undernourished and the ones presenting weight/height indices two $z$ scores above, overweight. Two Models of Multivariate Logistic Regression were built: one to identify stunting associated variables and the other for overweight associated variables.

Results: $5.2 \%$ stunting and 5.0\% overweight prevalence were determined. The variables of child's age group and the number of siblings were inversely associated to the two diseases in studies, in which to have two or more siblings and to be less than two years were risk factors for stunting, and to have two or more siblings and to be less than five years were protection factors for overweight.

Conclusions: the prevalence of the stunting and the overweight above the expected for the reference population justifies nutritional interventions.
\end{abstract}

Key words Nutritional status, Child, Malnutrition, Overweight, Child day care centers

\section{Resumo}

Objetivos: descrever os fatores associados ao estado nutricional de crianças assistidas em creches da Prefeitura do Município de São Paulo.

Métodos: foi selecionada amostra probabilística de crianças de 4 a 84 meses $(n=556)$, utilizando procedimento de amostragem por conglomerados. Adotou-se o padrão de referência do National Centre for Health Statistics, para os índices peso/idade, peso/estatura e estatura/idade, considerando-se em déficit nutricional as crianças que apresentaram esses índices dois escores $z$ abaixo do valor mediano da população de referência e sobrepeso com índice pesolestatura dois escores z acima. Foram construídos dois modelos de regressão logística multivariada para identificar quais as variáveis associadas com o déficit de estatura e o sobrepeso.

Resultados: encontrou-se 5,2\% de prevalência de déficit de estatura e 5,0\% de sobrepeso. As variáveis número de irmãos e faixa etária da criança se associaram inversamente com os dois agravos em estudo, onde ter dois ou mais irmãos e idade inferior a dois anos é fator de risco para déficit de estatura e ter dois ou mais irmãos e idade inferior a cinco anos é fator de proteção para sobrepeso.

Conclusões: verificou-se prevalência de déficit de estatura e sobrepeso acima do esperado para a população de referência, justificando intervenções nutricionais que contemplem esses dois agravos.

Palavras-chave Estado nutricional, Criança, Desnutrição, Sobrepeso, Creches 


\section{Introdução}

As creches públicas desempenham papel fundamental na vida das famílias de baixa renda no Brasil, pois possibilitam a participação da mulher no mercado de trabalho, aumentando a renda familiar, além de ter um papel importante na melhoria da condição de saúde das crianças que as freqüentam, uma vez que se associam à educação, ao cuidado básico de saúde, à alimentação e à higiene das crianças. 1,2

Vários estudos demonstram associação positiva entre a permanência das crianças em creches e seu estado nutricional, constatando diminuição dos déficits de peso e estatura, principalmente entre as crianças com maior tempo de permanência.1,3

Há, porém, alguns aspectos negativos em relação à saúde das crianças que freqüentam creches, pois esses ambientes podem constituir-se em fator de risco, tanto para o aumento da freqüência de episódios de doenças infecto-contagiosas, quanto para contração de doenças de maior gravidade, que podem comprometer o estado nutricional.4,5

Apesar dos aspectos negativos relatados em vários estudos, devemos considerar que esses efeitos podem ser minimizados com medidas preventivas apropriadas e que a utilização das creches por crianças em condições socioeconômicas menos favorecidas pode ser considerada uma das estratégias dos países subdesenvolvidos para garantir o crescimento e desenvolvimento das mesmas. As creches em áreas pobres podem ser utilizadas para realização de intervenções, a fim de promover a melhoria das condições de saúde e nutrição. 3,6

No entanto, essas intervenções, se freqüentes e não devidamente monitoradas, podem contribuir para o aumento da prevalência da obesidade, devendo ser, portanto, utilizadas com o acompanhamento nutricional adequado e ajustes individuais. ${ }^{7}$

No Município de São Paulo as creches públicas atendem 84.000 crianças entre 4 e 84 meses e são destinadas à população de baixa condição socioeconômica. Devido à escassez de dados sobre o estado nutricional dessas crianças, com amostra representativa de toda a rede municipal, o objetivo deste artigo é descrever os fatores associados ao estado nutricional de crianças assistidas em creches da Prefeitura do Município de São Paulo, a fim de subsidiar a formulação e/ou reformulação das ações nutricionais em creches públicas desse Município.

\section{Métodos}

Para o presente estudo foram utilizados dados do projeto temático "Nutrição e Saúde: uma abordagem integrada para a avaliação nutricional, desenvolvimento de alimentos para fins especiais e intervenção nutricional", que está sendo desenvolvido no Departamento de Nutrição da Faculdade de Saúde Pública (FSP) da Universidade de São Paulo (USP). Esse projeto coletou dados antropométricos, socioeconômicos e de morbidade, através de questionário previamente testado, de crianças de 4 a 84 meses de idade atendidas pelas creches da Prefeitura do Município de São Paulo. Foram realizadas três coletas de dados antropométricos para cada criança, com intervalos de aproximadamente seis meses entre si, nos anos de 1999 e 2000.

Foi selecionada uma amostra probabilística de creches, utilizando-se procedimento de amostragem por conglomerados em duas etapas. Na primeira etapa foram sorteadas 21 creches de um total de 718 . Para garantir a representatividade das regiões e das diversidades administrativas do município, as creches foram ordenadas para sorteio segundo regiões administrativas e tipo de administração (direta, indireta ou conveniada). Considerou-se também o número de crianças por creche. Creches menores foram reunidas para se obter uma distribuição o mais uniforme possível de crianças. As crianças a serem avaliadas nas creches

\section{Figura 1}

Número de crianças incluídas e perdidas na coorte, ao longo do seguimento de um ano, das creches do município de São Paulo. São Paulo, 2003.

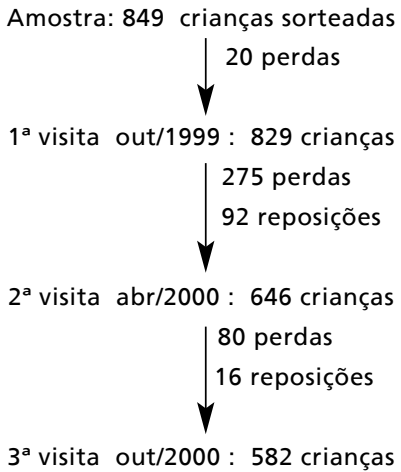


amostradas foram selecionadas por sorteio sistemático. A fração global de amostragem utilizada foi: $f \mathrm{i}=0,0098=20 \mathrm{Mi} / \sum M i \quad \mathrm{x} \mathrm{ci} / \mathrm{Mi}$, onde $M i=$ o número de crianças na creche $i$ e $c i=$ o número de crianças sorteadas em cada creche $i$.

A Figura 1 mostra as perdas e reposições das crianças incluídas na coorte ao longo do seguimento de um ano (1999/2000).

A fim de analisarmos a possibilidade de viés da amostra, comparamos as características das crianças da amostra final com as características das crianças que foram perdidas. Assim, vimos que, com exceção da variável idade da criança, as outras variáveis de interesse (socioeconômicas e antropométricas) não diferiram entre os dois grupos. Isso nos permite expandir os resultados obtidos para o total da população de crianças atendidas em creches públicas municipais da cidade de São Paulo.

Este artigo analisa os dados antropométricos coletados na segunda visita do projeto temático, com amostra inicial de 646 crianças, que resultou em 556 crianças depois das exclusões do banco de dados. Trata-se, portanto, de um estudo transversal com utilização de dados secundários.

Foram excluídas, de acordo com o critério de exclusão proposto pela Word Health Organization (WHO) ${ }^{8}$ as crianças classificadas em pelo menos uma das seguintes condições: índice estatura/idade inferior a cinco escores $\mathrm{z}$ e superior a três, índice peso/estatura inferior a quatro escores $\mathrm{z}$ e superior a cinco e índice peso/idade inferior a cinco escores $\mathrm{z}$ e superior a cinco do valor mediano da população de referência $(n=20)$, além daquelas de que não se tinham informações dos dados antropométricos $(\mathrm{n}=$ 26), das variáveis número de irmãos $(\mathrm{n}=1)$ e renda per capita $(\mathrm{n}=43)$.

Em relação às outras variáveis em estudo, que não se mostraram estatisticamente significantes na análise univariada, as crianças que tinham algum campo não preenchido não foram eliminadas, com o objetivo de não aumentar o número de perdas, e foi considerado um campo "sem informação" para cada uma dessas variáveis.

As medidas antropométricas de peso e estatura foram tomadas em duplicata, usando-se a média de ambas. O peso foi coletado utilizando-se balança microeletrônica portátil, marca Tanita ${ }^{\circledR}$, capacidade para $150 \mathrm{Kg}$ e precisão de $200 \mathrm{~g}$. As crianças utilizaram apenas uma peça leve (cueca ou calcinha) para tomada de peso e no caso de crianças menores, que usavam fraldas, estas foram retiradas. Crianças menores de dois anos foram pesadas no colo do entrevistador, cujo peso foi deduzido do peso total.

As crianças maiores de dois anos foram medidas em pé e descalças, utilizando-se estadiômetro vertical (precisão de $0,1 \mathrm{~cm}$ ), construído pelo Núcleo de Pesquisas Epidemiológicas em Nutrição e Saúde (NUPENS) da USP. Os menores de dois anos foram medidos deitados (tomada do comprimento), utilizando-se o infantômetro (Modelo ARTHAG).

Os pesquisadores foram treinados no Laboratório de Avaliação nutricional de populações (LANPOP) da FSP da USP para a padronização das medidas antropométricas e controle da variabilidade inter e intra-observadores seguindo as recomendações de Lohman et al. ${ }^{9}$ e Habicht et al. 10

Para análise dos dados antropométricos da população em estudo, utilizou-se como referência a tabela do National Center for Health Statistics (NCHS), recomendada pela WHO $^{8}$ para os índices antropométricos peso/idade, estatura/idade e peso/estatura. Consideraram-se em déficit nutricional todas as crianças que apresentaram esses índices dois escores $\mathrm{z}$ abaixo do valor mediano da população de referência, e com sobrepeso as crianças cujos índices peso/estatura estavam dois escores $\mathrm{z}$ acima do valor mediano.

O software Epi-info, versão 6.04c, foi utilizado para estruturação e composição do banco de dados. Foi realizada dupla digitação e posterior utilização do módulo validate para comparar as digitações e depurar o banco de dados final. Todas as fichas foram criticadas antes da digitação.

Foram construídos dois modelos para o cálculo de regressão logística: o primeiro para identificar quais as variáveis que tinham associação com o déficit nutricional e o segundo para identificar as que tinham associação com o sobrepeso.

Para analisar a influência das variáveis no déficit de estatura, considerou-se como variável dependente: $(y)=$ estatura/idade $(\geq-2 z=0 ;<-2 z=1)$ e as variáveis independentes: faixa etária da criança, sexo da criança, peso ao nascer, número de irmãos, infecções de vias aéreas superiores (IVAS), diarréia, renda familiar per capita, escolaridade materna, idade materna, situação marital (com ou sem companheiro). O índice estatura/idade foi utilizado para analisar a influência das variáveis no déficit nutricional por refletir déficit de longa duração. ${ }^{8}$ Para o Modelo de Regressão Logística Multivariada para sobrepeso, a variável dependente adotada foi $(\mathrm{y})=$ peso/estatura $(\leq 2 \mathrm{z}=0 ;>2 \mathrm{z}=1)$ e as variáveis independentes: faixa etária da criança, sexo da criança, peso ao nascer, número de irmãos, renda familiar per capita, escolaridade materna, idade materna, situação marital.

Para o processo de seleção das variáveis em estudo a serem incluídas na análise de Regressão 
Logística Multivariada, adotou-se nível crítico de significância $p \leq 0,20$ da análise univariada. As variáveis foram adicionadas no modelo, uma de cada vez, em ordem crescente do valor de $p$. Na análise de Regressão Logística Multivariada foi adotado nível de significância $p \leq 0,05$. Foram utilizados os pacotes estatísticos Epi-info v. 6.04c e MULTLR.

O estudo foi apreciado e aprovado pelo Comitê de Ética da Faculdade de Saúde Pública da
Universidade de São Paulo de acordo com os requisitos da Resolução 196/96.

\section{Resultados}

Foi avaliado um total de 556 crianças, sendo 278 $(50,0 \%)$ do sexo masculino. A distribuição foi homogênea segundo o sexo, em todas as faixas

Tabela 1

Distribuição de crianças atendidas em creches municipais segundo déficit de estatura e as variáveis em estudo. São Paulo, 2003.

\begin{tabular}{|c|c|c|c|c|c|c|c|}
\hline \multirow[t]{3}{*}{ Variáveis / Categorias } & \multicolumn{4}{|c|}{ Estado Nutricional } & \multicolumn{2}{|c|}{ Total } & \multirow[t]{3}{*}{$p$} \\
\hline & \multicolumn{2}{|c|}{ Sem déficit de estatura } & \multicolumn{2}{|c|}{ Com déficit de estatura } & \multirow[b]{2}{*}{ n (556) } & \multirow[b]{2}{*}{$\%$} & \\
\hline & n (527) & $\%$ & n (29) & $\%$ & & & \\
\hline \multicolumn{8}{|l|}{ Faixa etária da criança } \\
\hline$<2$ anos & 93 & 88,6 & 12 & 11,4 & 105 & 100,0 & 0,003 \\
\hline$\geq 2$ anos & 434 & 96,2 & 17 & 3,8 & 451 & 100,0 & \\
\hline \multicolumn{8}{|l|}{ Sexo da criança } \\
\hline Masculino & 262 & 94,2 & 16 & 5,8 & 278 & 100,0 & 0,703 \\
\hline Feminino & 265 & 95,3 & 13 & 4,7 & 278 & 100,0 & \\
\hline \multicolumn{8}{|l|}{ Peso ao nascer } \\
\hline$\geq 2500 \mathrm{~g}$ & 455 & 95,0 & 24 & 5,0 & 479 & 100,0 & 0,793 \\
\hline$<2500 \mathrm{~g}$ & 52 & 92,9 & 4 & 7,1 & 56 & 100,0 & \\
\hline Sem informação & 20 & 95,0 & 1 & 5,0 & 21 & 100,0 & \\
\hline \multicolumn{8}{|l|}{ Número de irmãos } \\
\hline$\leq 1$ & 316 & 97,5 & 8 & 2,5 & 324 & 100,0 & 0,001 \\
\hline 2 e mais & 211 & 90,9 & 21 & 9,1 & 232 & 100,0 & \\
\hline \multicolumn{8}{|l|}{ IVAS } \\
\hline Ausência & 340 & 95,0 & 18 & 5,0 & 358 & 100,0 & 0,540 \\
\hline Presença & 106 & 93,0 & 8 & 7,0 & 114 & 100,0 & \\
\hline Sem informação & 81 & 96,4 & 3 & 3,6 & 84 & 100,0 & \\
\hline \multicolumn{8}{|l|}{ Diarréia } \\
\hline Ausência & 383 & 95,0 & 20 & 5,0 & 403 & 100,0 & 0,333 \\
\hline Presença & 63 & 91,3 & 6 & 8,7 & 69 & 100,0 & \\
\hline Sem informação & 81 & 96,4 & 3 & 3,6 & 84 & 100,0 & \\
\hline \multicolumn{8}{|l|}{ Renda per capita (sm) } \\
\hline $0 \vdash 0,5$ & 175 & 91,1 & 17 & 8,9 & 192 & 100,0 & 0,009 \\
\hline 0,5 e mais & 352 & 96,7 & 12 & 3,3 & 364 & 100,0 & \\
\hline \multicolumn{8}{|l|}{ Escolaridade materna } \\
\hline $0-7$ anos & 295 & 95,2 & 15 & 4,8 & 310 & 100,0 & 0,853 \\
\hline 8 e mais & 185 & 94,4 & 11 & 5,6 & 196 & 100,0 & \\
\hline Sem informação & 47 & 94,0 & 3 & 6,0 & 50 & 100,0 & \\
\hline \multicolumn{8}{|l|}{ Idade materna } \\
\hline$<20$ anos & 16 & 94,1 & 1 & 5,9 & 17 & 100,0 & 0,927 \\
\hline $20 \mapsto 40$ anos & 436 & 95,0 & 23 & 5,0 & 459 & 100,0 & \\
\hline$>40$ anos & 22 & 95,7 & 1 & 4,3 & 23 & 100,0 & \\
\hline Sem informação & 53 & 93,0 & 4 & 7,0 & 57 & 100,0 & \\
\hline \multicolumn{8}{|l|}{ Situação marital } \\
\hline Com companheiro & 305 & 96,2 & 12 & 3,8 & 317 & 100,0 & 0,215 \\
\hline Sem companheiro & 167 & 92,8 & 13 & 7,2 & 180 & 100,0 & \\
\hline Sem informação & 55 & 93,2 & 4 & 6,8 & 59 & 100,0 & \\
\hline
\end{tabular}

IVAS = Infecções das Vias Aéreas Superiores; sm = salários mínimos de março de 2000. 
etárias em estudo e observou-se freqüência mais elevada de crianças na faixa etária entre dois e cinco $\operatorname{anos}(63,1 \%)$.

A média da renda familiar foi de 3,7 salários mínimos, sendo que $34,5 \%$ das crianças pertenciam a famílias com renda familiar abaixo de 0,5 salário mínimo. Apesar das creches municipais de São Paulo serem destinadas à população de baixa condição socioeconômica, com renda familiar máxima de quatro salários mínimos, no grupo estudado 35,8\% das crianças tinham renda familiar acima de quatro salários mínimos, observando-se famílias com renda de até $R$ \$ 5.000,00 reais (equivalente a 36,7 salários mínimos em março 2000).

Notou-se que $55,7 \%$ das mães tinham até sete anos de escolaridade, sendo que 16 mães eram analfabetas. Em 82,5\% dos casos as mães apresentaram idade entre 20 e 40 anos, sendo a média de 29 anos.

Em relação ao número de irmãos, $28,1 \%$ dos casos eram filhos únicos, 30,2\% tinham apenas um irmão e 41,7\% tinham dois irmãos ou mais. A média verificada foi de um irmão e o número máximo de nove.

Analisando-se os índices antropométricos,

Tabela 2

Distribuição de crianças atendidas em creches municipais segundo sobrepeso e as variáveis em estudo. São Paulo, 2003.

\begin{tabular}{|c|c|c|c|c|c|c|c|}
\hline \multirow[t]{3}{*}{ Variáveis / Categorias } & \multicolumn{4}{|c|}{ Estado Nutricional } & \multicolumn{2}{|c|}{ Total } & \multirow[t]{3}{*}{$p$} \\
\hline & \multicolumn{2}{|c|}{ Sem sobrepeso } & \multicolumn{2}{|c|}{ Com sobrepeso } & \multirow[b]{2}{*}{ n (556) } & \multirow[b]{2}{*}{$\%$} & \\
\hline & n (528) & $\%$ & n (28) & $\%$ & & & \\
\hline \multicolumn{8}{|l|}{ Faixa etária da criança } \\
\hline$<5$ anos & 437 & 95,8 & 19 & 4,2 & 456 & 100,0 & 0,080 \\
\hline$\geq 5$ anos & 91 & 91,0 & 9 & 9,0 & 100 & 100,0 & \\
\hline \multicolumn{8}{|l|}{ Sexo da criança } \\
\hline Masculino & 265 & 95,3 & 13 & 4,7 & 278 & 100,0 & 0,846 \\
\hline Feminino & 263 & 94,6 & 15 & 5,4 & 278 & 100,0 & \\
\hline \multicolumn{8}{|l|}{ Peso ao nascer } \\
\hline$\geq 2500 \mathrm{~g}$ & 453 & 94,6 & 26 & 5,4 & 479 & 100,0 & 0,481 \\
\hline$<2500 \mathrm{~g}$ & 54 & 96,4 & 2 & 3,6 & 56 & 100,0 & \\
\hline Sem informação & 21 & 100,0 & 0 & 0,0 & 21 & 100,0 & \\
\hline \multicolumn{8}{|l|}{ Número de irmãos } \\
\hline 0 & 143 & 91,7 & 13 & 8,3 & 156 & 100,0 & 0,081 \\
\hline 1 & 161 & 95,8 & 7 & 4,2 & 168 & 100,0 & \\
\hline 2 e mais & 224 & 96,6 & 8 & 3,4 & 232 & 100,0 & \\
\hline \multicolumn{8}{|l|}{ Renda per capita (sm) } \\
\hline $0,0 \vdash 0,5$ & 182 & 94,8 & 10 & 5,2 & 192 & 100,0 & 0,928 \\
\hline $0,5 \vdash 1,0$ & 177 & 94,7 & 10 & 5,3 & 187 & 100,0 & \\
\hline 1,0 e mais & 169 & 95,5 & 8 & 4,5 & 177 & 100,0 & \\
\hline \multicolumn{8}{|l|}{ Escolaridade materna } \\
\hline $0-7$ anos & 297 & 95,8 & 13 & 4,2 & 310 & 100,0 & 0,057 \\
\hline $8-10$ anos & 125 & 95,4 & 6 & 4,6 & 131 & 100,0 & \\
\hline 11 anos e mais & 58 & 89,2 & 7 & 10,8 & 65 & 100,0 & \\
\hline Sem informação & 48 & 96,0 & 2 & 4,0 & 50 & 100,0 & \\
\hline \multicolumn{8}{|l|}{ Idade materna } \\
\hline$<20$ anos & 16 & 94,1 & 1 & 5,9 & 17 & 100,0 & 0,222 \\
\hline $20 \mapsto 40$ anos & 436 & 95,0 & 23 & 5,0 & 459 & 100,0 & \\
\hline$>40$ anos & 20 & 87,0 & 3 & 13,0 & 23 & 100,0 & \\
\hline Sem informação & 56 & 98,2 & 1 & 1,8 & 57 & 100,0 & \\
\hline \multicolumn{8}{|l|}{ Situação marital } \\
\hline Com companheiro & 298 & 94,0 & 19 & 6,0 & 317 & 100,0 & 0,347 \\
\hline Sem companheiro & 172 & 95,6 & 8 & 4,4 & 180 & 100,0 & \\
\hline Sem informação & 58 & 98,3 & 1 & 1,7 & 59 & 100,0 & \\
\hline
\end{tabular}

sm = salários mínimos de março de 2000. 
Tabela 3

Medidas de Risco (Razão de chances- RC) e Intervalo de Confiança (IC95\%) para déficit de estatura segundo as variáveis número de irmãos e faixa etária da criança. São Paulo, 2003.

\begin{tabular}{lccccc}
\hline Variáveis & Categorias & $\mathbf{N}$ & RC não ajustada (IC95\%) & RC ajustada (IC95\%) & $\boldsymbol{p}^{*}$ * \\
\hline Número de irmãos & $\leq 1$ & 8 & 1 & 1 & 0,014 \\
& 2 e mais & 21 & $3,93(1,71<R C<9,04)$ & $2,93 \quad(1,20<R C<7,12)$ & 1 \\
Faixa etária da criança & $\geq 2$ anos & 17 & 12 & $3,29(1,52<R C<7,13)$ & $2,94 \quad(1,34<R C<6,47)$ \\
& $<2$ anos & 12 & 0,010 \\
\hline
\end{tabular}

* Modelo controlado por sexo da criança e renda familiar per capita; ** valor de p para tendência linear.

Tabela 4

Medidas de Risco (Razão de chances - RC) e Intervalo de Confiança (IC95\%) para sobrepeso segundo as variáveis número de irmãos e faixa etária da criança. São Paulo 2003.

\begin{tabular}{lccccc}
\hline Variáveis & Categorias & $\mathbf{N}$ & RC não ajustada (IC95\%) & RC ajustada (IC95\%) & $p^{* *}$ \\
\hline Número de irmãos & 0 & 13 & 1 & 1 & 0,034 \\
& 1 & 7 & $0,48(0,19<R C<1,32)$ & $0,41 \quad(0,16<R C<1,08)$ \\
& 2 e mais & 8 & $0,39(0,16<R C<0,97)$ & $0,28(0,11<R C<0,77)$ \\
Faixa etária da criança & $\geq 5$ anos & 9 & 1 & 1 \\
& $<2$ anos & 12 & $0,44(0,19<R C<1,00)$ & $0,39(0,17<R C<0,90)$ \\
\hline
\end{tabular}

* Modelo controlado por sexo da criança e renda familiar per capita; ** valor de p para tendência linear.

encontraram-se $5,2 \%$ de crianças com déficit de estatura, $3,1 \%$ com déficit de peso/idade, $0,9 \%$ com déficit de peso/estatura e $5,0 \%$ de crianças com sobrepeso.

$\mathrm{Na}$ análise univariada, os fatores que se associaram ao déficit de estatura foram: faixa etária da criança (<2 anos), número de irmãos $(\leq 1)$ e renda per capita $(<0,5$ salário mínimo). Nenhum fator associou-se ao sobrepeso na análise univariada, considerando-se nível crítico de significância $p$ $\leq 0,05$ (Tabelas 1 e 2).

As variáveis "número de irmãos" e "faixa etária da criança" foram as únicas que mantiveram associação estatística com os dois agravos estudados na análise de Regressão Logística apresentando, porém, relação inversa.

As crianças com dois irmãos ou mais, apresentaram maior risco de ter déficit de estatura, de quase três vezes $\left(\mathrm{RC}_{\text {ajustada }}=2,93\right)$, quando comparadas com as crianças sem irmãos e com apenas um irmão. Por outro lado, essa mesma variável apresentou fator de proteção para sobrepeso quando as crianças apresentaram dois irmãos ou mais $\left(\mathrm{RC}_{\text {ajustada }}=0,28\right)$, em relação as crianças que não tinham irmão (Tabelas 3 e 4).
Ao se analisar a variável "faixa etária da criança", nota-se que as crianças menores de dois anos apresentaram maior risco, em quase três vezes, de apresentar déficit de estatura $\left(\mathrm{RC}_{\text {ajustada }}=2,94\right)$ quando comparadas com as maiores de dois anos, e que ter idade inferior a cinco anos foi fator de proteção para sobrepeso $\left(\mathrm{RC}_{\text {ajustada }}=0,39\right)$, em relação às crianças com cinco anos ou mais (Tabelas 3 e 4).

\section{Discussão}

A prevalência de $5,2 \%$ crianças com déficit de estatura e 5,0\% de sobrepeso está acima do esperado quando comparada à da população de referência, que é de 2,3\%. A prevalência do déficit de peso/ estatura $(0,9 \%)$ foi inferior ao esperado, indicando que a forma de desnutrição caracterizada pelo emagrecimento extremo das crianças é rara nesse grupo.

Os resultados encontrados neste estudo, em relação ao tipo de déficit nutricional mais prevalente, são compatíveis com outros estudos brasileiros, demonstrando predomínio do déficit estatural e 
baixa prevalência de crianças com emagrecimento extremo. $2,11-14$

No Estado de São Paulo verificou-se situação semelhante, ou melhor, em relação ao déficit nutricional, ao encontrado nas crianças desse estudo, 2,11,12 porém as prevalências encontradas ainda são bem menores do que tem sido observado nas regiões Nordeste e Norte do Brasil. 15

Quando os dados desse estudo são analisados por faixa etária, nota-se claramente que entre as crianças menores de dois anos o déficit de estatura ainda é um problema de saúde relevante, atingindo prevalência de $11,4 \%$, o que justifica a continuidade de ações nutricionais que visem o combate desse agravo entre as crianças dessa faixa etária.

Outros estudos corroboram esses resultados. Silva e Sturion, 2 ao descreverem o estado nutricional de pré-escolares nos Centros Educacionais e Creches do Município de Piracicaba, São Paulo, entre crianças de 0 e 7 anos de idade, verificaram que é na faixa etária de 12 a 24 meses que se concentram as maiores proporções de desnutrição crônica. Batista Filho e Romani13 também encontraram maior prevalência de déficit estatural na faixa etária de 12 a 24 meses de idade.

Ao mesmo tempo em que o déficit estatural ainda é um agravo importante entre os menores de dois anos estudados, observa-se alta prevalência de sobrepeso entre as crianças maiores de cinco anos $(9,0 \%)$, situação que reflete a Transição Epidemiológica e Nutricional, pela qual o país está passando e que exige planejamento de intervenções nutricionais adequadas 14,16-18 que possam contemplar também a prevenção da obesidade entre as crianças institucionalizadas.

Gigante et al. 14 ao compararem as coortes de nascimento de 1982 e 1993, em Pelotas, constataram um aumento na prevalência de sobrepeso, cerca de duas vezes maior nas crianças nascidas em 1993, quando comparadas com aquelas de idade semelhante nascidas em 1982. Por outro lado, há uma diminuição na prevalência de déficit de estatura de quase $50 \%$, comparando as mesmas crianças nos dois períodos. Post et al., 19 ao analisarem as mesmas coortes, referem que a desnutrição está sendo substituída pela obesidade como o principal problema nutricional das crianças Pelotenses.

Entre os pré-escolares da rede pública do Município de Cosmópolis, São Paulo, em geral de baixo nível socioeconômico, o predomínio do sobrepeso foi oito vezes maior do que o da desnutrição (razão sobrepeso/déficit de peso). 12

A variável "número de irmãos" apresentou associação estatística com os dois agravos estudados na análise de Regressão Logística. Em relação ao déficit nutricional, a literatura corrobora o resultado verificado neste estudo. Guimarães et al. ${ }^{20}$ relatam que crianças cujas mães tiveram quatro ou mais filhos apresentaram chance 3,5 vezes maior de ter déficit de estatura quando comparadas com crianças cujas mães tiveram apenas um filho. Os dados da Pesquisa Nacional sobre saúde e Nutrição (PNSN) de 1989 também demonstraram que na região Sul (zona urbana) do Brasil, ter dois ou mais filhos menores de cinco anos implica um risco 4,3 vezes maior de retardo de crescimento em relação a ter apenas um filho. 21

O menor número de filhos por mulher significa melhores condições de cuidado, inclusive psicossocial. Ocorre, nesse caso, menor competição pelo tempo materno, fato mais relevante para as crianças menores, dependentes das mães para serem alimentadas e atendidas em relação à higiene, cuidados com a saúde e estimulação cognitiva. Ter poucos filhos pode também ser um facilitador para a entrada da mulher no mercado de trabalho, o que possibilita melhores condições de vida. Pelos dois caminhos, o impacto resultante do menor número de filhos tende a ser favorável à redução da prevenção da desnutrição. 22

As taxas de fecundidade vêm apresentando sensível e contínua queda no Brasil, o que fará com que as proles numerosas, até certo momento fator de risco para desnutrição, se tornem um problema decrescente em nosso meio. 23

Ao mesmo tempo em que a queda da taxa de fecundidade tem um impacto positivo em relação à desnutrição, o sobrepeso pode despontar como um agravo nutricional importante. Isso, mesmo nos estratos socioeconômicos mais desfavorecidos, caso se confirme, através de mais estudos, a associação do sobrepeso infantil com o menor número de irmãos. São escassos os estudos sobre obesidade infantil no Brasil, entretanto em estudo realizado em Belo Horizonte, com estudantes entre 6 e 18 anos, de escolas públicas, os autores também relatam maior prevalência de sobrepeso quando há menor número de irmãos. ${ }^{17}$

Vários estudos demonstram associação negativa entre renda per capita e desnutrição infantil,2,11,22 verificando-se que à medida que aumenta a renda, diminui sistematicamente a proporção de crianças desnutridas.

Essa associação pode ser constatada neste estudo, pois a prevalência de déficit de estatura variou de $3,3 \%$ a $8,9 \%$, entre as crianças de maior e menor renda, respectivamente. A variável "renda" mostrou associação com déficit de estatura na análise 
univariada, porém, com o ajuste de outras variáveis, o efeito da renda per capita sobre a variável dependente (déficit de estatura) perdeu a significância estatística e atenuou o efeito das outras variáveis: número de irmãos e faixa etária da criança.

Em relação ao sobrepeso, vários autores relatam prevalência maior entre as crianças de maior renda.11,24,25 No presente estudo, no entanto, a variável renda não apresentou associação com o sobrepeso, notando-se pequenas diferenças na prevalência encontrada entre as crianças das diversas faixas de renda. Neste grupo, a baixa renda não é fator de proteção para o sobrepeso infantil, diferentemente dos resultados encontrados por outros autores.

Monteiro, 26 estudando a prevalência de obesidade em países com diferentes níveis de riqueza, constatou que apenas as sociedades extremamente pobres parecem protegidas desse agravo. Os dados sugerem que o desenvolvimento econômico e a magnitude da obesidade não são fenômenos necessariamente relacionados, havendo países com níveis de riqueza semelhantes e prevalências muito distintas de obesidade. Nessas sociedades mais desenvolvidas, o risco de obesidade é função, essencialmente, do nível de educação e do acesso dos indivíduos à informação.

A escolaridade materna também vem sendo apontada como determinante do estado nutricional de crianças. 15,24,27 Os dados da Pesquisa Nacional sobre Saúde e Nutrição (PNSN) confirmaram existência de associação entre escolaridade materna e déficit de estatura, constatando que a escolaridade materna inferior a quatro anos associou-se com 4,3 vezes mais chance de déficit de estatura. 15

$\mathrm{O}$ fato da escolaridade materna persistir fortemente associada ao crescimento infantil, mesmo quando são semelhantes as condições socioeconômicas, está relacionado, provavelmente, com a importância que cuidados apropriados têm para a saúde e a nutrição da criança. ${ }^{15}$ Neste estudo, no entanto, a escolaridade materna não apresentou associação estatística significante com o déficit de estatura.

Em relação ao sobrepeso infantil, no presente estudo verifica-se, entre os filhos de mães com maior escolaridade, maior número de crianças com sobrepeso, sendo a prevalência de $10,8 \%$, enquanto que, para os filhos de mães com escolaridade entre 0 e 7 anos, a prevalência foi de 4,2\%. Esses resultados são compatíveis com os encontrados por Engstrom e Anjos, 24 que observaram o crescimento da prevalência de sobrepeso com o aumento da escolaridade materna. Porém, apesar da variável escolaridade materna, na análise univariada, ter apresentado nível crítico de significância, estipulado para o estudo $(p \leq 0,20)$, para ser incluída na análise de Regressão Logística Multivariada, o seu efeito foi eliminado quando incluído no modelo junto com as outras variáveis, não apresentando significância estatística.

Carvalhaes 22 conclui, em seu estudo, que a falta de companheiro associa-se com maior risco de desnutrição infantil. Relaciona esse fator mais aos aspectos definidores do acesso da criança, aos recursos necessários ao cuidado infantil (bens e serviços) do que à qualidade da atenção a ela dispensada.

Os resultados do presente estudo mostram maior número de crianças com déficit de estatura entre mães sem companheiro e maior número de crianças com sobrepeso entre mães com companheiro, embora sem confirmação desse resultado nos testes estatísticos aplicados.

Também não foi possível concluir sobre a interferência da idade materna com o risco de déficit de estatura e sobrepeso, pois a frequiência de casos foi pequena, provavelmente devido à baixa representatividade das faixas etárias de mães menores de 20 anos e maiores de 40 , sendo de $3,1 \%$ e $4,1 \%$, respectivamente.

Vários estudos têm evidenciado que condições da situação nutricional intra-uterina, refletidas pelo peso ao nascer, constituem determinantes importantes não só da sobrevivência infantil, como também, do estado nutricional nos primeiros anos de vida.22,27,28 No presente estudo, no entanto, o número de casos de crianças que nasceram abaixo de $2500 \mathrm{~g}$ e que apresentaram déficit de estatura foi muito pequeno (quatro casos), inviabilizando qualquer tipo de análise.

As infecções de vias aéreas superiores (IVAS) e diarréias neste estudo, não apresentaram significância estatística, apesar do número de casos de déficit de estatura ser maior entre os grupos que apresentaram esses dois agravos. Devemos considerar que foram analisadas informações sobre as freqüências desses agravos apenas no último mês anterior à pesquisa e que esse tipo de déficit (estatura/idade) reflete condição de má nutrição ou saúde inadequada em longo prazo. ${ }^{8}$ Considera-se que não é plausível biologicamente que uma infecção recente cause esse tipo de déficit. 27

\section{Considerações finais}

As crianças menores de dois anos foram as mais acometidas pelo déficit de estatura, como já verifi- 
cado em outros estudos, devendo haver, por isso, acompanhamento sistemático desse agravo nesse grupo.

Visto que o aumento da prevalência de sobrepeso ocorreu entre as crianças com cinco anos ou mais, justifica-se, também o monitoramento desse agravo em escolares do ensino fundamental.

O menor número de filhos pode ser uma estratégia importante para a redução do déficit estatural, porém são necessárias medidas de educação nutricional e estímulo de atividade física, para que, entre as famílias menores, o sobrepeso não venha despontar como um problema de saúde pública.

É importante salientar a necessidade de mais estudos que permitam a análise dos determinantes do sobrepeso infantil, uma vez que as pesquisas sugerem a possibilidade de crescimento desse agravo nessa população. Neste trabalho, contudo, algumas variáveis relevantes para o sobrepeso infantil, como o estado nutricional dos pais e os aspectos da relação mãe e filho, não foram coletadas, por falta de viabi-

\section{Referências}

1. Silva EMK. Avaliação do estado nutricional de crianças de creches públicas e crianças que recebem outros tipos de cuidado diurno no município de Embu, SP [tese doutorado]. São Paulo: Escola Paulista de Medicina da Universidade Federal de São Paulo; 1996.

2. Silva MV, Sturion GL. Freqüência à creche e outros condicionantes do estado nutricional infantil. Rev Nutr. 1998; 11: 58-68.

3. Taddei JA, Cannon MJ, Warner L, Souza P, Vitalle S, Palma D, Nóbrega F. Nutritional gains of underprivileged children attending a day care center in S. Paulo city, Brazil: a nine month follow-up study. Rev Bras Epidemiol. 2000; 3: 2937.

4. Benicio MHD, Cardoso MRA, Gouveia NC, Monteiro CA. Tendência secular da doença respiratória na infância na cidade de São Paulo (1984-1996). Rev Saúde Pública. 2000; 34 (Supl 6): 91-101.

5. Barros AJD. Freqüência a creches e morbidade comum na infância: evidência de associação na literatura e problemas de delineamento. Rev Saúde Pública. 1999; 33: 98-106.

6. Silva EMK, Miranda CT, Puccini RF, Nóbrega FJ. Day care centres as an institution for health promotion among needy children: an analytical study in São Paulo, Brazil. Public Health. 2000; 114: 385-8.

7. Uauy R, Albala C, Kain J. Obesity trends in Latin American: transiting from under- to overweight. J Nutr. 2001; 131: 893S-9S.

8. WHO (World Health Organization). Physical status: the use and interpretation of anthropometry. Geneva; 1995 (Technical Report Series, 854). lidade de custo e operacionalização.

As creches podem e devem promover intervenções nutricionais adequadas para a melhoria do estado nutricional, pois as crianças podem ser facilmente identificadas quando em risco nutricional e monitoradas. Para tanto, é necessária a implantação do Sistema de Vigilância Alimentar e Nutricional (SISVAN), que é um Sistema de informações, com coleta, processamento e análise contínua de dados, com o objetivo de monitorar o estado nutricional e alimentar. Esse Sistema pode ser facilmente implantado em creches municipais.

\section{Agradecimentos}

À Maria Regina Alves Cardoso. Departamento de Epidemiologia da Faculdade de Saúde Pública da Universidade de São Paulo. À Agência financiadora: Fundação de Amparo à Pesquisa do Estado de São Paulo (Fapesp) (Processo 98/08095-9).

9. Lohman TG, Roche AF, Martorrel R. Anthropometric standardization reference manual. Champaign, IL: Human Kinetics Books; 1988.

10. Habicth JP, Martorrel R, Yarbrough C, Malina RM, Klein RE. Height and weight standard for preschool children: how relevant are ethnic differences in growth potential? Lancet. 1974; 1: 611-5.

11. Monteiro CA, Conde WL. Tendência secular da desnutrição e da obesidade na infância na cidade de São Paulo (19741996). Rev Saúde Pública. 2000; 34 (Supl 6): 1-4.

12. Guimarães LV, Barros MBA. As diferenças de estado nutricional em pré-escolares de rede pública e a transição nutricional. J Pediatr. [Rio J] 2001; 77: 381-6.

13. Batista Filho M, Romani SAM. Alimentação, nutrição e saúde no Estado de Pernambuco. Recife: IMIP; 2002.

14. Gigante DP, Victora CG, Araújo CLP, Barros FC. Tendências no perfil nutricional das crianças nascidas em 1993 em Pelotas, Rio Grande do Sul, Brasil: análises longitudinais. Cad Saúde Publica. 2003; 19 (Suppl 1): S141-S7.

15. Monteiro CA, Benicio MHD, Freitas ICM. Melhoria em indicadores de saúde associados à pobreza no Brasil dos anos 90: descrição, causas e impacto sobre desigualdades regionais. São Paulo: NUPENS/USP; 1997.

16. Monteiro CA, Mondini L, Souza ALM, Popkin BM. Da desnutrição para a obesidade: a transição nutricional no Brasil. In: Monteiro CA, organizador. Velhos e novos males da saúde no Brasil: a evolução do país e de suas doenças. São Paulo: HUCITEC; 1995. p. 247-55. 
17. Oliveira ADB, Oliveira RG, Ribeiro RQ, Lamounier JA. Prevalência e fatores de risco de obesidade em estudantes de ensino médio e fundamental: o estudo de Belo Horizonte. In: Anais do Simpósio: Obesidade e Anemia Carencial na Adolescência; 2000 jun 8-9; Salvador, BA, Brasil. Rio de Janeiro: Instituto Danone; 2000. p. 245-6.

18. Batista Filho M, Rissin A. A transição nutricional no Brasil: tendências regionais e temporais. Cad Saúde Pública. 2003; 19 (Supl 1): 181-91.

19. Post CL, Victora CG, Barros FC, Horta BL, Guimarães PRV. Desnutrição e obesidade infantis em duas coortes de base populacional no Sul do Brasil: tendências e diferenciais. Cad Saúde Pública. 1996; 12 (Suppl 1): 49-57.

20. Guimarães LV, Latorre MRDO, Barros MBA. Fatores de risco para a ocorrência de déficit estatural em pré-escolares. Cad Saúde Pública. 1999; 15: 605-15.

21. Cardoso MAA. Cuidado infantil e desnutrição de pré-escolares: regiões Nordeste e Sul do Brasil [tese doutorado]. São Paulo: Faculdade de Saúde Pública da USP; 1995.

22. Carvalhaes MABL. Desnutrição e cuidado infantil: um estudo de casos e controle [tese doutorado]. São Paulo: Faculdade de Saúde Pública da Universidade de São Paulo; 1999.
23. Patarra NL. Mudanças na dinâmica demográfica. In: Monteiro CA, organizador. Velhos e novos males da saúde no Brasil: a evolução do país e de suas doenças. São Paulo: HUCITEC; 1995. p. 61-78.

24. Engstrom EM, Anjos LA. Relação entre o estado nutricional materno e sobrepeso nas crianças brasileiras. Rev Saúde Pública. 1996; 30: 233-9.

25. Corso ACT, Viteritte PL, Peres MA. Prevalência de sobrepeso e sua associação com a área de residência em crianças menores de seis anos de idade matriculadas em creches públicas de Florianópolis, Santa Catarina, Brasil. Rev Bras Epidemiol. 2004; 7: 201-9.

26. Monteiro CA. Epidemiologia da obesidade. In: Halpern A, Matos AFG, Suplicy HL, Mancini MC, Zanella MT. Obesidade. São Paulo: Lemos; 1998. p. 55-6.

27. Olinto MTA, Victora CG, Barros FC, Tomasi E. Determinantes da desnutrição infantil em uma população de baixa renda: um modelo de análise hierarquizada. Cad Saúde Pública. 1993; 9 (Supl): 14-27.

28. Monteiro CA. Evolução da nutrição infantil nos anos 90. In: Monteiro CA, organizador. Velhos e novos males da saúde no Brasil: a evolução do país e de suas doenças. São Paulo: HUCITEC; 1995. p. 375-92.

Recebido em 16 de junho de 2005

Versão final apresentada em 17 de agosto de 2006

Aprovado em 25 de agosto de 2006 